\title{
Análisis de esfuerzo de muestreo de himenópteros parasitoides en tres ambientes del este uruguayo
}

\section{Sampling effort analysis for hymenopteran parasitoids in three environments of Eastern Uruguay}

Castiglioni, Enrique (1); Perioto, Nelson W. (2); Lara, Rogéria I. R. (2); Burla, Juan P. (1); Arbulo, Natalia (1); Aldabe, Joaquín (1). (1) Centro Universitario Regional del Este (CURE), Universidad de la República (UdelaR), Rocha, Uruguay.

(2) APTA Ribeirão Preto, Laboratório de Bioecologia e Taxonomia de Parasitoides e Predadores, Ribeirão Preto, SP, Brasil.

Contacto: bbcastbb@gmail.com

RECIBIDO: $31 / 3 / 2017$ - APROBADO: 9/6/2017

\begin{abstract}
Resumen
Los himenópteros parasitoides brindan el servicio ecosistémico de control biológico de numerosas plagas agrícolas. Muchos de ellos son minúsculos y su separación e identificación son altamente trabajosas. Entre diciembre de 2014 y diciembre de 2015 se condujeron colectas quincenales con dos trampas Malaise en un área natural, una de pastoreo con ganado vacuno y una con ganadería vacuna con agricultura inverno-estival, en las cercanías de la ciudad de Castillos, Rocha, Uruguay. La separación de taxones colectados se realizó en el Centro Universitario Regional del Este (CURE) - Rocha y se identificaron las familias de los himenópteros parasitoides en Ribeirão Preto, Brasil. Se colectaron 36.718 parasitoides de 31 familias de Hymenoptera parasitoides. Con datos transformados a abundancia proporcional en la muestra se construyeron sets considerando una $(\mathrm{N}=72)$ o dos quincenas $(\mathrm{N}=144)$ y se realizó un análisis multivariado de acumulación de familias (perMANOVA). Considerando la mitad (una quincena) o la totalidad (dos quincenas) de las fechas, indistintamente, es posible ajustar el esfuerzo de muestreo para disminuir la frecuencia y/o los meses de muestreo, sin pérdida significativa en el hallazgo de nuevas familias. Este estudio contribuye a mantener la eficiencia y validez del trabajo y a reducir costos en recursos humanos y materiales. Palabras clave: Abundancia, diversidad, Hymenoptera parasitica, trampas Malaise.
\end{abstract}

\begin{abstract}
Hymenopteran parasitoids offer an ecosystem service as biological control of agricultural pests. Many of these parasitoids are tiny insects and, therefore, their screening and identification are highly complex. Fortnightly sampling, between December 2014 and December 2015, was conducted with two Malaise traps in three areas: natural area, cattle grazing pastures and cattle grazing with winter-summer agriculture, near Castillos, Rocha, Uruguay. Taxa were screened at the Centro Universitario Regional del Este (CURE) - Rocha and parasitoids identification, at family level of the order Hymenoptera, was made in Ribeirão Preto, São Paulo State, Brazil. In total, 36.718 parasitoid individuals from 31 Hymenoptera families were collected. Data sets were created with information transformed to proportional abundance in the sample: considering one $(\mathrm{N}=72)$ or two $(\mathrm{N}=144)$ fortnights, and a family accumulation multivariate analysis was applied (perMANOVA). Considering half (one fortnight) or total (two fortnights) number of sampling dates, indistinctly, it is possible to adjust sampling effort in order to decrease the sampling frequency or dates, without a significant reduction on the recovering of new families. Thus, this is a contribution to maintain the recovery efficiency and the value of the study, while helping to reduce costs in material and human resources.

Keywords: Abundance, diversity, Hymenoptera parasitica, Malaise traps.
\end{abstract}

\section{Introducción}

La expansión e intensificación agrícolas constituyen una de las principales causas de la disminución global de la biodiversidad (Tilman, et al., 2001). Esto tiene como consecuencia la disminución del funcionamiento ecosistémico $\mathrm{y}$, por consiguiente, de los niveles de servicios que brindan al ser humano, como el equilibrio de poblaciones, reciclaje de nutrientes, entre otros (Naeem, et al., 1999, Loreau, et al., 2001, Balvanera, et al., 2006). La intervención antrópica en los agroecosistemas altera la estructura y el funcionamiento de los ecosistemas originales, cambian los índices y la eficiencia del reciclaje de nutrientes, se altera el flujo de energía y aumenta la dependencia de los insumos externos (Gliessman, et al., 
2007). Por esas razones, los agroecosistemas son considerados sistemas poco estables y poco diversificados. Dentro de las amenazas globales, las actividades agropecuarias representan algunas de las principales acciones negativas para la diversidad biológica, en tanto determinan una simplificación de la estructura del ambiente en áreas extensas, sustituyendo la diversidad natural por un limitado número de especies vegetales y animales domesticados (Altieri, et al., 2003). En Uruguay, la expansión e intensificación de la agricultura han determinado cambios importantes en el uso de la tierra, que generan nuevos desafíos para una gestión sostenible de la biodiversidad (CLAES, et al., 2008; DINAMA, 2010). El tradicional sistema mixto agrícola-ganadero del país se ha ido sustituyendo por extensiones agrícolas puras (Castiglioni, et al., 2008), disminuyendo de forma consistente la proporción del área agrícola total que entra a la fase pastoril como pradera (DIEA, 2011).

El grado de conocimiento de la biodiversidad de Uruguay es muy irregular en los diversos grupos objeto de estudio (CLAES, et al., 2008) y la información acerca del impacto de los diversos usos de la tierra sobre la biodiversidad nativa es muy escasa (DINAMA, 2010). De hecho, algunos grupos de artrópodos que ocurren en agroecosistemas y proporcionan valiosos servicios ecosistémicos, como el control biológico de plagas, han sido poco estudiados. Entre ellos se destacan los insectos parasitoides, que poseen características intermedias entre los predadores y los parásitos: como los primeros, matan al hospedero que atacan y, como muchos parásitos, requieren de solo un hospedero, dentro o sobre cuyo cuerpo se desarrollan (Eggleton y Belshaw, 1992; Basso, 2009). Los cambios en los ecosistemas pueden afectar la riqueza y la efectividad de las comunidades naturales de los parasitoides (Marino y Landis, 1996).

Los himenópteros parasitoides comprenden aproximadamente el $75 \%$ del número estimado de especies de parasitoides (Belshaw, et al., 2003) y operan por vía natural limitando de forma aún no bien cuantificada las poblaciones de numerosos insectos perjudiciales, incluso en gran parte de los agroecosistemas (Bentancourt y Scatoni, 2001; Perioto, et al., 2004).

Para estudios de abundancia y diversidad de himenópteros parasitoides, la literatura refiere al empleo de diversos tipos de trampas (Lara, et al., 2015; Sobczak y Vasconcellos, 2015). Las más ampliamente utilizadas son las trampas Malaise (Dall'Oglio, et al., 2003; Sperber, et al., 2004; Alencar, et al., 2007; Feitosa, et al., 2007; Santos, 2007; Mazón y Bordera, 2008; Oliveira, et al., 2011; Costa Jr, et al., 2014), debido a su mayor eficiencia en la captura de himenópteros voladores de diversos tamaños, tanto en el día como en la noche, así como su bajo costo y facilidad de operación, ya que solo se cambia el frasco colector en cada fecha programada. Son trampas de interceptación de vuelo, que se constituyen de una estructura de carpa de tejido fino suspendida por estacas. La captura es eficiente, dado que la parte inferior de tejido negro dificulta su percepción por parte de los insectos interceptados, mientras que la parte superior del tejido es blanca y promueve el flujo de estos hacia el frasco de captura situado en la parte superior (Güler, 2008; Sarmiento, 2006; Townes, 1972).

La alta eficiencia de esta técnica de muestreo involucra, sin embargo, una demorada y dificultosa tarea en el laboratorio para la separación de taxones colectados a campo y su posterior identificación. Por lo tanto, se requiere una planificada elaboración de las frecuencias y períodos de muestreo, con el objetivo de que resulte compatible un adecuado y eficiente muestro de la diversidad de taxones de interés, con la disponibilidad de recursos humanos y materiales en las posteriores etapas de separación e identificación. Con el objetivo de realizar aportes para futuros estudios de diversidad de Hymenoptera parasitica, en este trabajo se comparan esfuerzos de muestreo con trampas Malaise, en el período de un año, en tres sitios con sistemas productivos de diferente grado de intervención antrópica en las cercanías de la ciudad de Castillos, departamento de Rocha, Uruguay.

\section{Materiales y Métodos}

Se realizaron colectas quincenales con trampas Malaise (Townes, 1972) (Figura 1) en el período comprendido entre el 29 de diciembre de 2014 y el 15 de diciembre de 2015, en tres sitios con un gradiente de intensidad de uso del suelo, en las cercanías de la ciudad de Castillos, Rocha, Uruguay. Las áreas fueron a) CN: Área de campo natural, con baja intensidad de

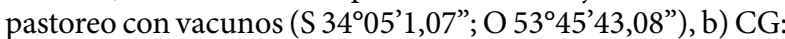
Área en producción bajo pastoreo de ganado vacuno y lanar (S 3405'26,80"; O 5352'14,40"), c) AG: Sistema integrado de producción ganadera con agricultura inverno-estival

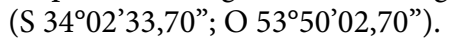

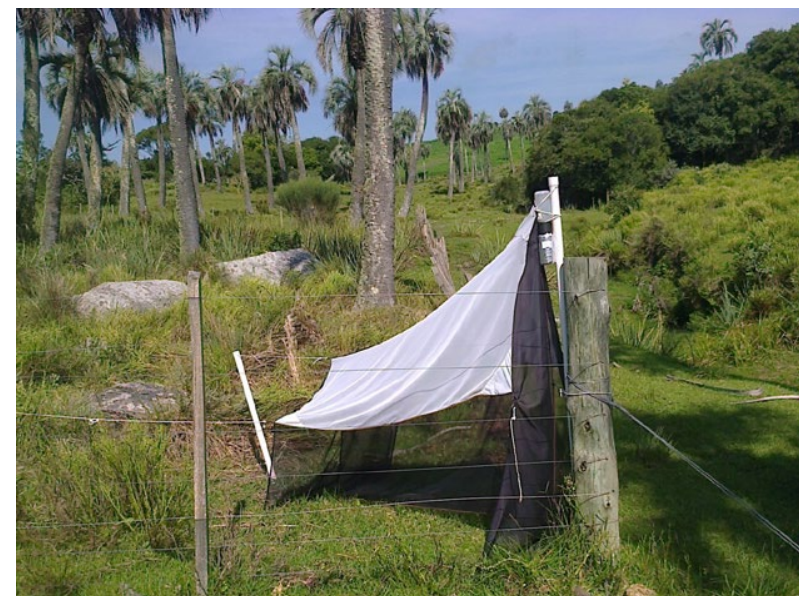

Figura 1. Trampa Malaise modelo Townes (1972).

Estas áreas se seleccionaron dentro de una matriz general, buscando condiciones ambientales originales y climáticas similares. La distancia geográfica plana se situó en $10052 \mathrm{~m}$ entre los sitios a y b, $6319 \mathrm{~m}$ entre b y c, y $4549 \mathrm{~m}$ entre los sitios a y c. En cada uno de los tres sitios se colocaron dos trampas Malaise, con una distancia mínima de $100 \mathrm{~m}$ entre ambas, y se caracterizó la vegetación predominante mediante el método de transectos. En las tres formaciones vegetales se realizó un censo florístico en parcelas de 20×20 metros, ubicadas dentro del área de acción de las trampas (Figura 2).

La separación, catalogación y cuantificación de los insectos contenidos en cada muestra se realizó en el Laboratorio de Biología Animal del Centro Universitario Regional del Este, en la ciudad de Rocha. La identificación de los himenópteros parasitoides fue realizada en el Laboratório de Bioecologia e Taxonomia de Parasitoides e Predadores, APTA, Ribeirão Preto, SP, Brasil.

Para el análisis de esfuerzo de muestreo se utilizaron los resultados de presencia/ausencia de familias en la muestra y 


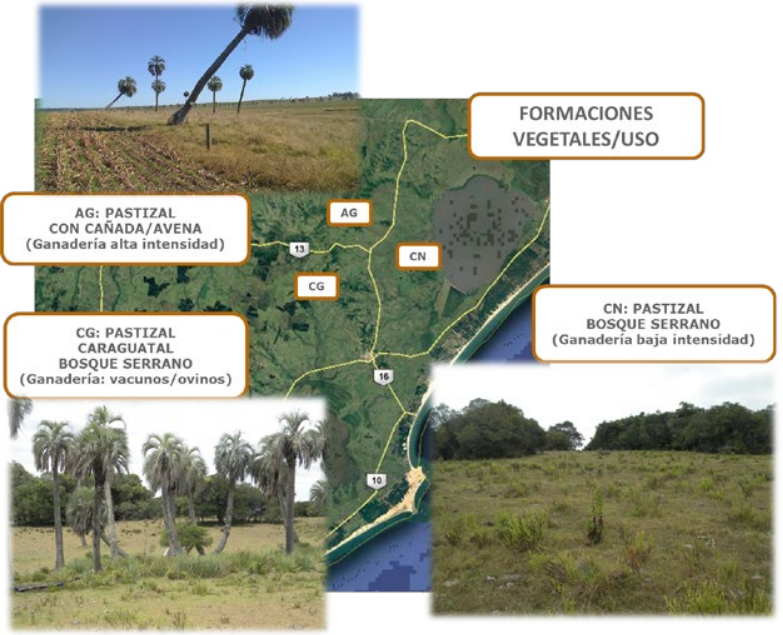

Figura 2. Localización y descripción de formaciones vegetales y sistema de manejo (uso) de los sitios de muestreo.

se realizaron curvas de acumulación de familias para set de datos con distinto esfuerzo de muestreo. Las mismas fueron construidas seleccionando las muestras de manera aleatoria a través de permutaciones. Se construyeron tres sets de datos: a) set $1=24$ colectas: dos quincenas $(\mathrm{N}=144)$; b) set $2=12$ colectas quincena $1(\mathrm{~N}=72)$; c) set $3=12$ colectas quincena $2(\mathrm{~N}=72)$. Para evaluar diferencias en composición de especies y abundancia entre los sets de datos se realizó un análisis multivariado PERMANOVA (Modelos multivariados no-paramétricos), considerando los tres sitios simultáneamente y los sitios por separado, donde las familias y sus abundancias son la variable de respuesta y el set de datos es la variable explicativa. Dado que los sets presentaron diferencias en el esfuerzo de muestreo, se consideró la proporción de individuos de cada familia en relación al total de individuos colectados. Los análisis y curvas de acumulación fueron realizados con Software R (3.3.1), Paquete «Vegan» (R Core Team, 2016).

\section{Resultados}

En el período total de un año de muestreos quincenales se colectaron 36.718 ejemplares pertenecientes a 31 familias de Hymenoptera Parasitica (Tabla 1).

El rango de abundancia de representantes de las distintas familias varió entre un mínimo de dos individuos (Embolemidae) y un máximo de 9057 (Mymaridae) en el total de las fechas de colecta.

La caracterización vegetal de los sitios reflejó una menor diversidad de especies y formaciones vegetales en el sitio con ganadería y agricultura (AG), en comparación con los sitios que no tienen fase agrícola (CN y CG) (Tabla 2). En ese sistema, que es el más intensificado (agrícola-ganadero), la formación vegetal predominante es el pastizal, con un menor número total de especies, principalmente herbáceas. En los sitios ganaderos hay una mayor diversidad vegetal, debido a que además de las especies del pastizal hay un número importante de arbóreas y de otras formas de vida (epífitas, trepadoras, arbustivas, suculentas) correspondientes a las formaciones vegetales bosque serrano (CN y CG) y caraguatal (CG).

Cuando se analizaron solamente las colectas de las primeras o segundas quincenas, en lugar de la totalidad de las muestras quincenales, las acumulaciones totales de familias fueron de 29 y 28 para las primeras y segundas quincenas,

\begin{tabular}{|l|c|}
\hline \multicolumn{1}{|c|}{ Familias } & \multicolumn{1}{|c|}{$\begin{array}{c}\text { Rango de abundancia } \\
\text { (total de ejemplares colectados/familia) }\end{array}$} \\
\hline $\begin{array}{l}\text { Mymaridae, Platygastridae, Eulophidae, Braconidae, Ichneumonidae, } \\
\text { Encyrtidae, Trichogrammatidae, Ceraphronidae }\end{array}$ & $>1000$ a 10000 \\
$\begin{array}{l}\text { Diapriidae, Figitidae, Aphelinidae, Pteromalidae, Chalcididae, Bethylidae, } \\
\text { Eupelmidae, Dryinidae, Signiphoridae, Eurytomidae }\end{array}$ & $>100$ a 1000 \\
$\begin{array}{l}\text { Torymidae, Agaonidae, Evaniidae, Tanaostigmatidae, Eucharitidae, } \\
\text { Megaspilidae, Heloridae, Gasteruptiidae, Perilampidae, Proctotrupidae, } \\
\text { Chrysididae, Leucospidae, Embolemidae }\end{array}$ & $>1$ a 100 \\
\hline
\end{tabular}

Tabla 1. Familias de himenópteros parasitoides colectados en un año de muestreos quincenales, según grado de abundancia, en tres sitios de la región de Castillos, Rocha.

\begin{tabular}{|c|c|c|c|c|c|}
\hline & & \multicolumn{3}{|c|}{ Número de especies vegetales según forma de vida } & \multirow[b]{2}{*}{ Total } \\
\hline & Formaciones vegetales & Herbáceas & Árboles/arbóreas & Otras (epífitas, etc.) & \\
\hline $\mathrm{CN}$ - Área natural & Bosque serrano/ pastizal & 26 & 10 & 21 & 57 \\
\hline CG - Área ganadera & $\begin{array}{l}\text { Bosque serrano/ } \\
\text { pastizal/ Caraguatal }\end{array}$ & 29 & 8 & 13 & 50 \\
\hline AG - Área agrícola-ganadera & Pastizal con cañada & 17 & 1 & 5 & 23 \\
\hline
\end{tabular}

Tabla 2. Caracterización vegetal de los sitios (CN, CG y AG) según formaciones vegetales, formas de vida y número de especies. 
respectivamente, incluyendo los tres sitios (Tabla 3). De modo que cuando el esfuerzo de muestreo se reduce a la mitad, en promedio, se estaría perdiendo la oportunidad de encontrar entre dos y tres familias de las registradas en este estudio.

\begin{tabular}{|c|c|c|c|}
\hline \multirow{2}{*}{} & \multicolumn{3}{|c|}{$\begin{array}{c}\text { Número máximo de familias } \\
\text { colectadas según quincenas } \\
\text { consideradas de colecta }\end{array}$} \\
\cline { 2 - 4 } & $\mathrm{Q} 1+2$ & $\mathrm{Q} 1$ & $\mathrm{Q} 2$ \\
\hline $\mathrm{CN}+\mathrm{CG}+\mathrm{AG}$ & 31 & 29 & 28 \\
\hline $\mathrm{CN}$ & 30 & 28 & 27 \\
$\mathrm{CG}$ & 28 & 28 & 25 \\
$\mathrm{AG}$ & 24 & 24 & 23 \\
\hline
\end{tabular}

Tabla 3. Máximo de familias colectadas con todas las muestras quincenales $(\mathrm{Q} 1+2)$, las muestras de las primeras quincenas (Q1) y las muestras de las segundas quincenas (Q2) en los tres sitios (CN, CG y AG) y por sitio individual.

El número total de familias recolectadas presentó una correspondencia con la heterogeneidad vegetal de cada localidad. A pesar de tratarse de tres sitios con matriz de pastizal nativo, las formaciones vegetales caracterizadas para cada uno de ellos mostraron un número decreciente de especies vegetales en el orden: área natural, área ganadera y área agrícola ganadera ( $\mathrm{Ta}-$ bla 2). Ese orden decreciente también se observó en el número total de familias de parasitoides capturadas (Tabla 3).

En relación a la estacionalidad de la abundancia de parasitoides, los mayores números de individuos por fecha fueron recolectados en primavera y verano, en tanto que el invierno fue la estación de colecta del menor número de individuos en todos los sitios (Figura 3).

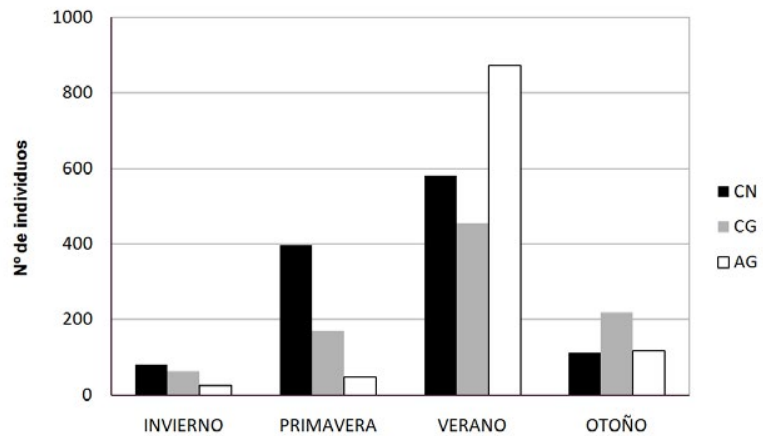

Figura 3. Abundancia de parasitoides colectados por sitio y por estación del año.

El análisis de acumulación de familias presenta tendencias similares tanto al incluir todas las colectas quincenales como al reducir los sets de muestras a la mitad, con las colectas realizadas en cada una de las quincenas solamente. Esta tendencia se observó analizando cada sitio en forma independiente (Figura 4) y realizando el análisis del total de las capturas agrupadas en los tres sitios. Si bien el número total de familias que se colectan es diferente en cada caso, la pendiente de las curvas siempre muestra una tendencia similar (considerando el total o la mitad de las fechas de muestreo y con los tres sitios o cada uno individualmente). Se verifica una rápida acumulación de familias en los primeros muestreos y una drástica disminución de la tasa de acumulación de nuevas familias poco después de la mitad del período de colectas.
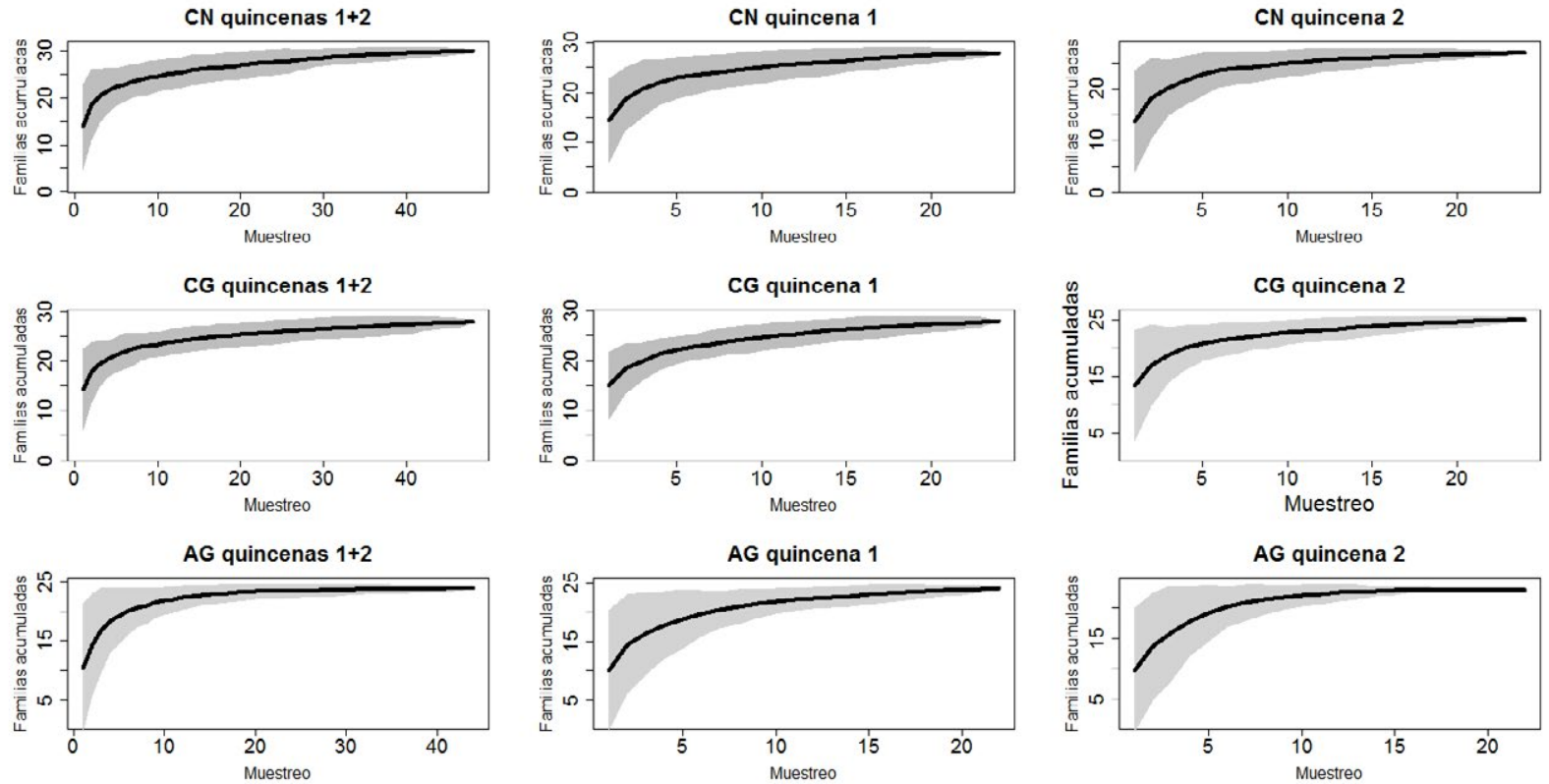

Figura 4. Curvas de acumulación de familias de himenópteros parasitoides en los tres sitios (CN, CG y AG) en los muestreos de ambas quincenas (Quincenas 1+2), de las primeras quincenas (Quincena 1) y de las segundas quincenas (Quincena 2). 


\begin{tabular}{|c|c|c|c|c|}
\hline & N & F Model & R2 & P $>$ F \\
\hline CN + CG + AG & 279 & 0,65463 & 0,00470 & 0,790 \\
CN & 95 & 0,16714 & 0,00358 & 1,000 \\
CG & 95 & 0,28006 & 0,00599 & 0,997 \\
AG & 95 & 0,16648 & 0,00390 & 1,000 \\
\hline
\end{tabular}

Tabla 4. Número de observaciones (N), Valor F (F Model), Coeficiente de determinación (R2) y significancia (P>F) del análisis PERMANOVA con 999 permutaciones, considerando los tres sitios en conjunto y cada sitio por separado.

Cuando la acumulación de familias en los tres sitios se estudió considerando los sets 2 y 3 , conformados por las muestras colectadas en las primeras y segundas quincenas, respectivamente, la disminución del número total de familias recuperadas ( 29 y 27 familias totales representadas de las 31 totales, respectivamente) no resulta estadísticamente significativa (Tabla 4).

Este análisis resulta válido también para las comparaciones de los muestreos de las primeras y segundas quincenas por separado y de ambas quincenas juntas, tomando en consideración las colectas de cada sitio en forma independiente.

\section{Discusión}

El total de familias (31) en las que se distribuyeron los ejemplares colectados corresponde aproximadamente al 70\% de las familias de himenópteros parasitoides conocidas para el Neotrópico (Fernández y Sharkey, 2006). Si bien para este grupo de insectos no existen inventarios realizados en la región de estudio, particularmente con este tipo de trampas, tal proporción de representación de las familias presentes en el Neotrópico confirma la alta eficiencia de las trampas Malaise para la colecta de himenópteros parasitoides, citada en la literatura regional revisada.

En términos comparativos, en el análisis de ambas frecuencias de muestreo (tanto si se realiza uno como dos muestreos quincenales por mes), se mantiene la tendencia a encontrar una menor abundancia de familias en el sitio AG (agrícola-ganadero), en el cual se recupera el menor número de familias totales. Esto podría explicarse por el hecho de que, al incluir agricultura, este sitio constituye el de mayor intervención antrópica de los tres estudiados y, por lo tanto, de menor disponibilidad de recursos. La recuperación de un menor número de familias de parasitoides en el sitio AG coincide con la menor diversidad vegetal verificada en este sitio, representado por el menor número de especies totales listadas en la caracterización vegetal efectuada (Tabla 2). Esto coincide con la teoría general de que los ambientes más simplificados en su constitución vegetal cuentan con menor diversidad de artrópodos (Gliessman, 2001; Altieri, 2004).

Por otra parte, los análisis estadísticos indican que se podría bajar el esfuerzo de muestreo reduciendo su frecuencia a la mitad, realizando solamente una colecta quincenal en cada mes (Tabla 3), ya que si bien existe una reducción en el número de familias halladas, esta no resulta significativa (Tabla 4). Esto significa un aporte importante para la planificación de estudios de esta naturaleza cuando existen recursos humanos y/o materiales limitados.
Lawton et al. (1998) destacan que la proporción de «morfoespecies» que no resulta posible identificar a nivel de especie y el número de horas de especialistas requeridos para la separación y el procesamiento de muestras aumentan en forma dramática con la abundancia de organismos de tamaño minúsculo; por consiguiente, los inventarios de organismos de pequeño tamaño, como los himenópteros parasitoides, demandan un considerable esfuerzo.

En términos descriptivos, se podría interpretar cuál fue el «costo» incurrido en número de fechas adicionales que, de acuerdo al análisis realizado, no hubieran sido necesarias para mantener la probabilidad de encontrar la última familia colectada, en promedio, para cada caso. Este análisis, realizado sobre los resultados obtenidos, propone la alternativa de haber suspendido los muestreos al momento de encontrar la última nueva familia (obtenida de los promedios resultantes de las curvas de acumulación de familias) y, por ese motivo, no haber destinado esfuerzo a los muestreos que no reportaron nuevos hallazgos. Para los casos analizados, cuando se considera la información global obtenida en los tres sitios, con la inclusión del total de muestras quincenales se podría haber acumulado la totalidad de las familias, en promedio, con aproximadamente el $80 \%$ de las fechas de muestreo efectivamente realizadas. Con una frecuencia de muestreo menor, esto es, una colecta por quincena (o en el caso de estudiar solamente un sitio), hubiera sido necesario mantener, en promedio, el $90 \%$ de las colectas efectivamente realizadas para garantizar no perder la última familia acumulada.

Este análisis hipotético, realizado con resultados vistos, subestima el costo del esfuerzo de muestreo, ya que indica que ese sería el número de fechas necesarias para encontrar la última familia. Pero esto no valoriza el costo global de las últimas colectas, que son las menos eficientes en términos de adicionar una nueva familia. Si se pudiera estimar cuál fue el «costo en número de colectas» que tuvo pasar de la «penúltima nueva familia» a la «última nueva familia» hallada, se tendría una idea aproximada de cuánto es necesario invertir en recursos para la obtención de esa «última nueva familia» (Tabla 5).

En la medida en que hay un mayor número de sitios o una mayor frecuencia de muestreos aumenta la eficiencia del muestreo en términos de representación de la abundancia de familias del lugar. Pero, al mismo tiempo, los sitios difieren en diversidad y abundancia de parasitoides, por lo cual resulta difícil obtener una respuesta matemática que indique el esfuerzo de muestreo ideal.

Debido a que un mayor esfuerzo de muestreo resulta en un incremento de salidas de campo, de recursos humanos y de recursos materiales destinados a la tarea de separación e identificación de ejemplares colectados, es importante 


\begin{tabular}{|c|c|c|c|c|c|c|c|}
\hline \multirow{2}{*}{} & \multicolumn{6}{|c|}{$\begin{array}{c}\text { Número de muestreos necesarios (en el total) hasta encontrar, } \\
\text { en promedio, la «penúltima nueva familia» }\end{array}$} \\
\cline { 2 - 9 } & \multicolumn{2}{|c|}{ Q1+2 } & \multicolumn{2}{c|}{ Q1 } & \multicolumn{2}{c|}{ Q2 } & \\
\cline { 2 - 9 } & Colectas & $\%$ & Colectas & $\%$ & Colectas & $\%$ & Promedio \\
\hline CN + CG + AG & $68 / 140$ & 48,6 & $23 / 70$ & 32,9 & $28 / 70$ & 40,0 & 40,5 \\
\hline CN & $28 / 48$ & 58,3 & $16 / 24$ & 66,7 & $13 / 24$ & 54,2 & 59,7 \\
CG & $29 / 48$ & 60,4 & $16 / 24$ & 66,7 & $13 / 24$ & 54,2 & 60,4 \\
\hline AG & $14 / 44$ & 31,8 & $13 / 22$ & 59,1 & $9 / 22$ & 40,9 & 43,9 \\
\hline Promedio/sitio & & 50,1 & & 64,2 & & 49,7 & \\
\hline
\end{tabular}

Tabla 5. «Costo adicional» promedio, en número fechas de muestreo, para hallar la «última nueva familia» con todas las quincenas $(\mathrm{Q} 1+2)$, las primeras $(\mathrm{Q} 1)$ y las segundas $(\mathrm{Q} 2)$ quincenas, en el conjunto de los tres sitios y en cada sitio.

contar con elementos para definir de una forma equilibrada la inversión en función de los objetivos y de los beneficios esperados de este tipo de estudios. El ejercicio que se presenta pone de manifiesto, de una forma gráfica, el beneficio de las primeras colectas y el alto costo de las últimas, en términos de las nuevas familias que se logra colectar.

A partir de estas consideraciones, el análisis realizado con los resultados obtenidos permite sugerir que la precisión de la representación de la composición de las familias de parasitoides que se obtiene con capturas en el tiempo con dos trampas Malaise por sitio de estudio podría mantenerse con una disminución en la frecuencia o en el período de colectas. Para no perder una representación precisa de las familias que ocurren hay un escaso margen de ajuste en el esfuerzo de muestreo, ya que no podría reducirse mucho más que al $80-90 \%$ de las colectas, según el número de sitios considerados. No obstante, de acuerdo a la significación del análisis estadístico realizado, es posible establecer que la reducción a la mitad de la frecuencia de colectas o en el período de muestreo permite realizar trabajos similares con recursos limitados sin perder información significativa.

En función de la estacionalidad de colecta de individuos encontrada en este estudio se podría sugerir restringir el período de muestreos a la primavera y al verano, cubriendo de esa manera los períodos de mayor actividad de los artrópodos y, por lo tanto, de mayor captura de ejemplares. En caso de contar con recursos suficientes podrían mantenerse también las colectas en otoño, considerando que la menor eficiencia de capturas ocurre en el invierno. El invierno, debido a la menor recuperación de ejemplares consecuente con las temperaturas más bajas, es la estación en la que menos se justifican las colectas. De esta forma se podría reducir el período de muestreo de los doce a unos ocho meses, aproximadamente. Este razonamiento concuerda con los períodos de muestreo que se emplean en colectas asociadas a cultivos anuales, sitios específicos o artrópodos con períodos de mayor actividad, cuyos estudios abarcan solo de uno a pocos meses (Dorfey, et al., 2011; Lara, et al., 2015; Fernandes, et al., 2014; Fraser, et al., 2007; Perioto, et al., 2005) o cuando el objetivo es determinar composición, abundancia o frecuencias relativas comparativas entre distintos ambientes (Marchiori, 2014). Por el contrario, si el objetivo es caracterizar con la mayor precisión y fidelidad la composición de familias o especies de un sitio, aun a escalas locales (Sääksjärvi, et al., 2004) y sobre todo en ambientes donde, por sus condiciones, la riqueza de especies es intrínsecamente baja (Várkony y Roslin, 2013), es necesaria una considerable inversión en número de colectas, asumiendo los mayores costos que implica esta decisión.

\section{Reconocimientos}

A los Sres. Walter Branaa, Fernando Ramos, Mario Cardoso, Ing. Agr. Agustín Piñeyrúa, por poner amablemente a disposición los sitios de estudio. Al Instituto Nacional de Investigación Agropecuaria - INIA Uruguay, por el financiamiento del Proyecto FPTA No 312 en el marco de la Convocatoria 2012.

\section{Referencias}

Alencar, I.D.C.C., Fraga, B., Tavares, M.T. y Azevedo C.O., 2007. Perfil da fauna de vespas parasitóides (Insecta, Hymenoptera) em uma área de Mata Atlântica do Parque Estadual de Pedra Azul, Domingos Martins, Espírito Santo, Brasil. En: Arquivos do Instituto Biológico, 74(2), pp.111-114.

Altieri, M.A., 2004. Agroecologia: a dinâmica produtiva da agricultura sustentável. Síntese Universitária. 4.ed. Porto Alegre: Ed. da UFRGS.

Altieri, M. A., Silva, E. N. y Nicholls, C. I., 2003. O papel da biodiversidade no manejo de pragas. Ribeirão Preto: Holos.

Balvanera, P., Pfisterer, A.B., Buchmann, N., He, J-S., Nakashizuka, T., Raffaelli D. y Schmid, B., 2006. Quantifying the evidence for biodiversity effects on ecosystem functioning and services. En: Ecology Letters, 9, pp.1146-1156.

Basso, C., 2009. Estructura de las comunidades de parasitoides. En: Basso, C. y Grille, G., eds., 2009. Relaciones entre organismos en los sistemas hospederos-parasitoidessimbiontes. Montevideo: Facultad de Agronomía. pp.9-26.

Belshaw, R., Grafen, A. y Quicke, D.L.J., 2003. Inferring life history from ovipositor morphology in parasitoid wasps using phylogenetic regression and discriminant analysis. En: Zoological Journal of the Linnean Society, 139, pp.213-228. 
Bentancourt, C.M. y Scatoni, I.B., 2001. Enemigos naturales. Manual ilustrado para la agricultura y la forestación. Montevideo: Editorial Hemisferio Sur.

Castiglioni, E., Ribeiro, A., Silva, H., Pereyra, C. y Martino, M., 2008. Algunas interrogantes y respuestas para el manejo de plagas en los sistemas agrícolas intensificados. En: Cangüé, 30, pp.25-29.

CLAES, PNUMA y Uruguay. Ministerio de Vivienda, Ordenamiento Territorial y Medio Ambiente. Dirección Nacional De Medio Ambiente, 2008. GEO Uruguay, 2008: informe del estado del ambiente. Montevideo: Mosca.

Costa Júnior, D.P. da., Oliveira, M.T., Nunes, J.F., Zampieron, S.L.M. y Peixoto, M.L., 2014. Avaliação da diversidade de insetos da Ordem Hymenoptera do Parque Nacional da Serra da Canastra (MG), coletados com armadilhas Malaise. En: Ciência et Praxis, 7(13), pp.21-26.

Dall'Oglio, O.T., Zanuncio, J.C., Freitas, F.A. de y Pinto, R., 2003. Himenópteros parasitóides coletados em povoamento de Eucalyptus grandis e mata nativa em Ipaba, Estado de Minas Gerais. En: Ciência Florestal, 13(1), pp.123-129.

DIEA, 2011. Encuesta agrícola primavera 2010 [En línea]. Montevideo: DIEA. [Consulta: 21 de febrero de 2012]. Disponible en: www.mgap.gub.uy

DINAMA, 2010. IV Informe nacional al convenio sobre la diversidad biológica. Montevideo: DINAMA.

Dorfey, C., Schoeninger, K. y Köhler, A., 2011. Levantamento das famílias de himenópteros parasitoides associados ao cultivo de tabaco (Nicotiana tabacum L.) em Santa Cruz do Sul e Lagoão, Rio Grande do Sul, Brasil. En: Arquivos do Instituto Biológico, 78(3), pp.449-451.

Eggleton, P. y Belshaw, R., 1992. Insect parasitoids: an evolutionary overview [En línea]. En: Biological Sciences, 337(1279), pp.1-20. [Consulta: 9 de marzo de 2017] Disponible en: http://www.jstor.org/stable/57087

Feitosa, M.C.B., Querino, R.B. y Henriques, A.L., 2007. Perfil da fauna de vespas parasitóides (Insecta: Hymenoptera) em reserva florestal na Amazônia, Amazonas, Brasil. En: Entomotropica, 22(1), pp.37-43.

Fernandes, D.R.R., Guimarães, J.A., Araujo, E.L., Lara, R.I.R. y Perioto, N.W., 2014. Survey of the Hymenoptera fauna in a "Caatinga» area in the State of Rio Grande do Norte, Northeastern Brazil. En: EntomoBrasilis, 7(3), pp.211-215.

Fernández, F. y Sharkey, M.J., 2006. Sistemática de los himenópteros de la región Neotropical: estado del conocimiento y perspectivas. En: Fernández, F. y Sharkey, M.J., eds., 2006. Introducción a los Hymenoptera de la región neotropical. Bogotá: Sociedad Colombiana de Entomología y Universidad Nacional de Colombia. pp.7-35.

Fraser, S.E.M., Dytham, C. y Mayhew, P.J., 2007. Determinants of parasitoid abundance and diversity in woodland habitats. En: Journal of Applied Ecology, 44, pp.352-361.

Gliessman, S.R., Rosado-May, F.J., Guadarrama-Zugasti, C., Jedlicka, J., Cohn, A., Mendez, V.E., Cohen, R., Trujillo, L., Bacon, C. y Jaffe, R., 2007. Agroecología: promoviendo una transición hacia la sostenibilidad. En: Ecosistemas, 16(1), pp.13-23.

Gliessman, S.R., 2001. Diversidade e estabilidade do agroecossistema. En: Gliessman, S.R., coord., 2001. Agroecologia: processos ecológicos em agricultura sustentável. 2. ed. Porto Alegre: Ed. UFRGS, pp.437-474. Güler, Y., 2008. Malaise traps. En: Mellifera, 8, pp.26-32. Lara, R.I.R., Fernandes, D.R.R., Versuti, D.R., Tango M.F. de A. y Perioto, N.W., 2015. Sampling and diversity of Hymenoptera (Insecta) in an orange orchard/Brazilian Savannah fragment interface. En: EntomoBrasilis, 8(1), pp.51-57.

Lawton J.H., Bignell D.E., Bolton B., Bloemers G.F., Eggleton R. y Hammond, R.M., 1998. Biodiversity inventories, indicator taxa and effects of habitat modification in tropical forest. En: Nature, 391, pp.72-76.

Loreau, M., Naeem, S., Inchausti, P., Bengtsson, J., Grime, J.P. y Hector, A., 2001. Biodiversity and ecosystem functioning: current knowledge and future challenges. En: Science, 294, pp.804-808.

Marchiori, C.H., 2014. Diversity of parasitoids collected in an area of forest and pastures in southern of Goiás, Brazil. En: Journal of Biology, 2(1), pp.14-17.

Marino P.C. y Landis, D.A., 1996. Effect of landscape structure on parasitoid diversity in agroecosystems. En: Ecological Applications, 6, pp.276-284.

Mazón, M. y Bordera, S., 2008. Effectiveness of two sampling methods used for collecting Ichneumonidae (Hymenoptera) in the Cabañeros National Park (Spain). En: European Journal of Entomology, 105, pp.879-888.

Naeem, S., Chapin, F.S., Costanza, R., Ehrlich, P.R., Golley, F.B., Hooper, D.U., Lawton, J.H., O.Neill, R.V., Mooney, H.A., Sala, O.E., Symstad, A.J. y Tilman, D., 1999. Biodiversity and ecosystem functioning: maintaining natural life support processes. En: Issues in Ecology, 4, pp.1-12.

Oliveira, R.G.M.G. de, Berti Filho, E., Peres Filho, O., Sales, F. da P. y Cunha, J.A.C. da, 2014. Diversity of hymenopteran parasitoids (Hymenoptera: Chalcididae) associated with teak (Tectona grandis) forests. En: Advances in Forestry Science, 1(2), pp.59-64.

Perioto, N.W., Lara, R.I.R., Selegatto, A. y Luciano, E.S., 2004. Himenópteros parasitóides (Insecta, Hymenoptera) coletados em cultura do café Coffea arabica L. (Rubiaceae) em Ribeirão Preto-SP, Brasil. En: Arquivos do Instituto Biológico, 71(1), pp.41-44.

Perioto, N.W., Lara R.I.R. y Selegatto, A., 2005. Himenópteros parasitóides da Mata Atlântica. II. Núcleo Grajaúna, Rio Verde da Estação Ecológica Juréia-Itatins, Iguape-SP, Brasil. En: Arquivos do Instituto Biológico, 72(1), pp.81-85.

$\mathrm{R}$ Core Team, 2016. $R$ : A language and environment for statistical computing [En línea]. Vienna: R Foundation for Statistical Computing. [Consulta: 8 de marzo de 2017] Disponible en: http://www.R-project.org.

Sääksjärvi, I.E., Haataja, S., Neuvonen, S., Gauld, I.D., Jussila, R., Salo, J. y Marmol Burgos, A., 2004. High local species richness of parasitic wasps (Hymenoptera: Ichneumonidae; Pimplinae and Rhyssinae) from the lowland rainforests of Peruvian Amazonia. En: Ecological Entomology, 29, pp.735-743.

Santos, P.S., 2007. Diversidade de himenópteros parasitóides em áreas de mata-de-cipó e cafezais em Vitória da Conquista-BA. Vitória da Conquista: UESB. (Disertación de Maestría).

Sarmiento, C.E., 2006. Familia Rhopalosomatidae. En: Fernández, F. y Sharkey, M.J., eds., 2006. Introducción de la Hymenoptera de la región neotropical. Bogotá: Sociedad 
Colombiana de Entomología y Unioversidad Nacional de Colombia. pp.517-520.

Sperber, F.C., Nakayama, K., Valverde, M.J. y Neves, F. de S., 2004. Tree species richness and density affect parasitoid diversity in cacao agroforestry. En: Basic and Applied Ecology, 5, pp.241-25.

Sobczak, J.F. y Vasconcellos Neto, J., 2015. Famílias de himenópteros parasitoides na Serra do Japi, Jundiaí, São Paulo, Brasil. En: Arquivos do Instituto Biológico, 82, pp.1-4.
Tilman, D., Fargione, J., Wolff, B., D’Antonio, C., Dobson, A. y Howarth, R., 2001. Forecasting agriculturally driven global environmental change. En: Science, 292, pp.281-284.

Townes, H., 1972. A light-weight Malaise trap. En: Entomological News, 83, pp.239-247.

Várkonyi, G. y Roslin, T., 2013. Freezing cold yet diverse: dissecting a high-Arctic parasitoid community associated with Lepidoptera hosts. En: The Canadian Entomologist, 145, pp.193-218. 